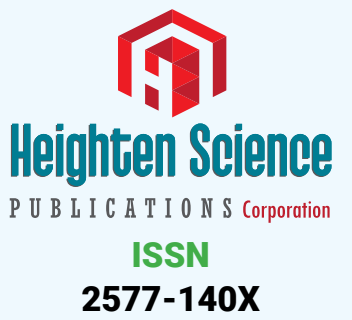

Address for Correspondence: William J Faulkner, Cincinnati Eye Institute, 1945 CEI Dr, Blue Ash, OH 45242, USA, Email:

Wfaulkner@cincinnatieye.com

Submitted: 01 November 2017

Approved: 20 December 2017

Published: 22 December 2017

Copyright: @2017 Faulkner WJ. This is an open access article distributed under the Creative Commons Attribution License, which permits unrestricted use, distribution, and reproduction in any medium, provided the original work is properly cited
Review Article

\section{The Role of Omega-3 Essential Fatty Acids in Dry Eye Disease}

\author{
William J Faulkner* \\ Cincinnati Eye Institute, 1945 CEI Dr, Blue Ash, OH 45242, USA
}

One of every four visits to eye care professionals is for dry eye disease which affects an estimated 7-34\% of Americans [1]. Knowledge regarding etiology and treatments has advanced exponentially in the last 20 years. As recently as 1997, in the mega textbook Cornea, it was stated "The mechanism for lacrimal gland dysfunction in this condition is unclear" [2]. A recent study entitled "The Dry Eye Workshop ll Report", defines dry eye as "a multifactorial disease of the ocular surface characterized by a loss of homeostasis of the tear film and accompanied by ocular symptoms in which tear film instability and hyperosmolarity, ocular surface inflammation, and damage and neurosensory abnormalities play etiological roles" [3]. A 2003 study regarding the morbidity ratings of dry eye showed utility assessments for moderate and severe dry eye were parallel to historical reports for more severe (class Ill/ IV) angina [4]. The tear film is responsible for the greatest optical power of any ocular surface, since the greatest change in the index of refraction occurs between the air and the tear film [5] Double pass retinal imaging shows increased light scatter in dry eye patients which can decrease retinal image quality 20-40\% [5]. Ophthalmologists now understand the critical necessity to normalize the ocular surface prior to cataract surgery; otherwise, they risk an unhappy patient with a less than ideal visual result. Increased use of electronic devices, such as smart phones, tablets, and computers, by youth in the last ten years has resulted in a broader age range of dry eye patients, to now include adolescents and teens. A study of urban school age children showed that $8.2 \%$ had dry eye (vs $2.8 \%$ in a rural group), presumably due to high incidence of smart phone use. ${ }^{6}$ In fact, with cessation of phone use for four weeks, dry eye resolved completely in $100 \%$ of subjects [6]. Only two FDA approved dry eye medications are available: cyclosporine ophthalmic emulsion, $0.05 \%$, (approved in 2003) and lifitegrast, 5\%, (approved in 2016), both of which require chronic treatment. The focus of this discussion involves an emerging paradigm in treating ocular surface disease: a nutraceutical containing omega-3 essential fatty acids.

Dry eye disease may be broadly characterized as aqueous deficient (quantity) or evaporative (quality). In one large study, $86 \%$ of patients with dry eye disease had an evaporative component due to meibomian gland disease, (MGD) [7]. The Meibomian glands secrete meibum, the oil stabilizing the tear film, essential for a normal tear break up time and a moist cornea. MGD is characterized by terminal duct obstruction and/or qualitative/ quantitative changes in glandular secretion. Clinical signs include foam formation at the lid margin due to break down of lipids from soaps to free fatty acids (saponification), a toothpaste-like secretion upon attempted gland expressionalso seen by meibomography, and a rapid tear break up time (TBUT).

Dietary patterns present for hundreds or thousands of years have changed in recent times. Essential fatty acids, only available through ingested food, fall into two major groups, Omega- 3 and Omega- 6 fatty acids, and are a requirement for multiple 
biological processes in the body. The ideal ratio in the body of Omega- $3 / 6$ is $1 / 1$ but with contemporary diets rich in animal fats, vegetable oils and processed foods, a common ratio is 1:20 or higher [8]. Key omega-3s including DHA (Docosahexaenoic acid) and EPA (Eicosapentaenoic Acid) are found in fish, flaxseed, walnuts, and avocados. They foster mental acuity, a healthy nervous system, immunity, regular heart rhythm, healthy heart growth, and reduction in inflammation, blood clots, serum triglycerides, and LDL cholesterol [9].

Among the early work (2000) supporting the efficacy of Omega-3's for dry eye, Carol Boerner, MD, followed 200 patients on 2,000 mg of flaxseed oil for three years. Symptoms improved in 85\% [10]. An Association for Research in Vision and Ophthalmology (ARVO) presentation in 2003 by Trivedi, Sana, Gilbard, et al. summarized the Harvard Women's Health Study involving 39,876 patients. Results showed that the more tuna fish consumed, the lower the risk of dry eye: 2-4 servings/ week cut risk by $18 \%$ (compared to less than 2), and 5-6 servings/ week reduced risk by $66 \%$. Not mentioned was the increased risk of mercury poisoning. In 2007 Athena Papas, DMD, et al. from Tufts University presented data at ARVO showed that utilizing an omega-3 supplement containing fish oil, flaxseed oil and vitamin E (Thera-tears Nutrition), resulted in significant reduction in symptoms of dry eye and dry mouth associated with Sjogren's syndrome (61 patients). Another study presented at ARVO in 2012 and sponsored by Science Based Health analyzed administration of omega-3 and gamma linolenic acid (Hydro Eye) for 6 months in 38 postmenopausal women. Improvements were noted in patient's symptoms (ocular surface disease index) and impression cytology inflammatory biomarkers, but no improvements were noted in corneal staining or tear breakup time.

A comparative study of three omega-3 preparations was reported by Frank Bucci, et al., at the American Academy of Ophthalmology meeting, 2011. This multicenter blinded randomized 3 month clinical study of 60 patients compared three oral supplements: PRN Dry Eye Omega Benefits, Nature made and Thera-tears [11]. Red blood cell membrane saturation index of Omega-3, EPA and DHA were measured [11]. The PRN preparation achieved desirable therapeutic levels of $8 \%$ after 1 month of use in $44 \%$ of patients, up from $4 \%$ at baseline; (measured by a simple finger stick blood test: Omega Quant, Sioux Falls, SD) [11]. Average values at 3 months were PRN 7.75\%, Nature made 6.6\%, and Thera-tears 5.9\% [11].

All Omega-3 preparations are not created equal, relative to quality, purity and efficacy. One important difference is the chemical form finalized by the manufacturing process. The most pristine natural source of EPA and DHA is from pelagic fish in the Tasman Sea. Unfortunately, virtually all commercially obtained fish oil is contaminated by mercury and carcinogens. Processing requires the use of alcohol or ethyl ester to purify this mix, but two undesirable sequelae ensue. The human body is poorly able to tolerate this foreign chemical alcohol leading to "fishy" side effects (dyspepsia) and perhaps more importantly, poor systemic absorption. For example a pill alleging $500 \mathrm{mg}$ of omega-3 in the ethyl ester form may only deliver 200mg. Furthermore, some OTC fish oil preparations in a water filled foam cup completely dissolve the cup bottom within several minutes. In order to remove the alcohol portion from the fish oil, a time consuming re-esterification process converts the product to its' original triglyceride form. This has proven to yield superior absorption [12]. The desirable value of $8 \%$ is known to be cardio-protective. Achieving a significant serum level was noted in a study over 20 years ago to reduce the risk of sudden death by myocardial infarction by $90 \%$ [13]. Depending on a person's weight and diet, an average dosage of 2 grams EPA and DHA daily for 2-3 months will likely achieve the desired level. Although flaxseed oil has some benefit due to abundant Omega-3's, it also contains alpha linoleic acid. ALA is partially metabolized to arachidonic acid, a pro-inflammatory product. 
Even with incomplete understanding of their mechanism of action, Omega-3 essential fatty acids were recommended as first line therapy by both the Dry Eye Workshop study group in 2007 and the International Workshop on Meibomian Gland Dysfunction in 2011 [14,15]. MGD is associated with altered lipid composition leading to increased tear evaporation and elevated tear osmolarity and subsequent ocular surface damage, epithelial cell desiccation, apoptosis and symptoms of burning and discomfort. High quality Omega-3 supplementation increases levels of unsaturated fatty acids in meibomian secretions which in a liquid state at room temperature prevent the blockage and stagnation within meibomian orifices. Thus tear film evaporation decreases, tear breakup time increases, and dry eye signs and symptoms improve. Additional benefits are decreased inflammation and increased tear secretion (by elevating CAMP).

An important feature of dry eye is inflammation and this condition, when associated with Omega-3 deficiency, can be linked to multiple other systemic illnesses. Many patients in the western world with dry eye also have age-related macular degeneration. In Japan these two conditions are virtually nonexistent due to a diet rich in Omega-3 EFA's. Cardiovascular studies have shown strong benefits linking omega-3 ingestion with reduced risk of cardiovascular disease $[16,17]$. These benefits include increased arterial elasticity, decreased erythrocyte "stickiness", decreased arrhythmias, decreased inflammation and decreased serum triglycerides. Young and middle aged patients with MGD have higher cholesterol levels than controls of similar age without MGD [18]. A 2016 study found that two large servings of oily fish / week reduced the risk of diabetic retinopathy by 48\% [19]. Recent research has identified a new class of signaling molecules called resolvins. Derived from DHA and EPA, these compounds are necessary components of the endogenous system which acts as the "off-switch" for the normal inflammatory response [20].

Perhaps the most thorough and widely read study on the efficacy of omega-3's appeared in Cornea, Sept., 2016, and analyzed the effects of a re-esterified triglyceride form in 105 patients [21]. This was a multicenter, prospective, interventional, placebo controlled, double masked study [21]. Subjects took 4 soft gels daily containing a total of $1680 \mathrm{mg}$ of EPA/560mg of DHA (Dry Eye Omega benefits, Physicians Recommended Nutraceuticals) or a control of $3136 \mathrm{mg}$ of linoleic acid daily for 12 weeks [21]. The conclusion was a statistically significant improvement in each of five parameters measured: Ocular Surface Disease Index score (patient's symptomatology), tear osmolarity, MMP-9 (an inflammatory marker), omega-3 serum index levels, and tear break up time [21].

Recent advances in knowledge regarding omega-3 essentials fatty acids will improve patient care both in dry eye disease and the rest of medicine. Two prescription oral omega-3 preparations are presently available: Lovaza (Omega-3-ethyl esters) and Vascepa (icosapent ethyl), a re-esterified triglyceride. The great majority of OTC omegas-3's are in the less desirable ethyl ester or alcohol form. A 2006 publication stated omega-3 deficiency or omeganemia was responsible for 96,000 deaths a yr. making it the sixth leading preventable cause of death in the United States [22]. Regarding dry eye and multiple other conditions, the happier category of patients are those with mild-moderate disease who become asymptomatic with treatment [22]. All patients begin at stage one; it is more difficult and less satisfying to treat advanced disease.

Finally at least two myths persisting about omega 3 essential fatty acids should be dispelled. First, patients on omega-3's EFA's are more prone to bleeding. This is true for most OTC brands due to the persistence of chemicals used to eliminate containments and pollutants. When these chemical are removed, the re-esterified triglyceride form is both digestible and not associated with hematologic abnormalities. Second, one article linked Omega-3's to prostate cancer [23]. This of course made national headlines 
and the nightly news. Prior studies involving over 500,000 subjects have shown a reduction in prostate cancer. The study was seriously flawed in many ways and was later retracted [24]. Unfortunately, bad information was falsely sensationalized, and the correction never made the front page.

Dry eye has a high impact on quality of life due to discomfort and/or visual disability. In a typical eye care practice, dry eye is the single most common complaint for patients seeking care. In both Dry Eye Workshop Study (DEWS) I \& II reports from 2007 and 2017 respectively, nutritional therapy is listed as first line treatment for even mild dry eye. Evidence for benefits of nutritional supplementation continues to mount. Chinnery, et al. found that Omega-3 supplementation imparts neuroprotective effects in the cornea sub-basal plexus which correlate with improvement in tear osmolarity [25]. In another study, rosacea patients showed improvement in symptoms, TBUT, Schirmer score and Meibomian gland score (MGS) with six months of Omega 3 supplementation [26]. An office-based study involving 1,419 patients in Spain found multiple benefits in dry eye sufferers utilizing a supplement that contained a combination of Omega-3 fatty acids, vitamins, minerals, and antioxidants [27]. A systematic review of fifteen independent studies involving 2,591 patients who used supplements with varying amounts of Omega-3 and Omega-6 EFA's was published in March, 2017 [28]. Subjective symptom improvement was noted in seven of the studies and TBUT significantly increased in nine of the studies [28]. One reason for the variable results is the variation in levels of Omegas in each supplement. While the AREDS II study failed to show statistical benefit for adding omega 3 EFA's, it was most likely because the less desirable form (ethyl ester) and a suboptimal dose of 1 gram, (350 mg DHA and 650mg EPA), were used [29]. The data is convincing that supplementation with high quality Omega-3 essential fatty acids (re-esterified triglyceride), in the proper dosage, is a starting point for treating this frustrating and potentially debilitating condition.

\section{References}

1. Murphy, John J. Dry Eye Inflammation: A Discussion with Dr. Michael Lemp, MD Linx.com. 2017.

2. Cornea, Vol II, Krachmer, Mannis, Holland. 1997; 647.

3. Sullivan DA, Daniel Nelson J, Jennifer Craig P, Esen Akpek K, Dimitri Azar T, et al. TFOS DEWS II Introduction. 2017; 15: 269-275. Ref.: https://goo.gl/A6QsFp

4. Schiffman RM, Walt JG, Jacobsen G, Doyle JJ, Lebovics G, et al. Utility assessment among patients with dry eye disease. Ophthalmology. 2003; 110: 1412-1419. Ref.: https://goo.gl/P7Dexj

5. Farid M. The contribution of the tear film to vision and ocular comfort. Eyeworld. September, 2016: 6.

6. Moon JH, Kim KW, Moon NJ. Smartphone use in a risk factor for pediatric dry eye disease according to region and age. a case control study. BMC Ophthalmol. 2016; 16: 188. https://goo.gl/bUbcHc

7. Lemp MA, Crews LA, Bron AJ, Foulks GN, Sullivan BD. Distribution of Aqueous deficient and Evaporative Dry Eye in a clinic Based patient Cohort: A retrospective Study. Cornea. 2012; 31: 472479. Ref.: https://goo.gl/ywiKyj

8. Simopoulos AP. Evolutionary aspects of diet, essential fatty acids and cardiovascular disease. Eur Heart J Supplements. 2001. Ref.: https://goo.gl/t7VxEh

9. Tribole E. The Ultimate Omega-3 Diet.

10. Boerner CF. Dry Eye successfully treated with oral flaxseed oil. Ocular Surgery News. 2000.

11. Bucci F, Kislan T. A Comparison of Omega-3 RBC Saturation for PRN-Dry Eye Omega Benefits V. Theratears Nutrition V. Nature Made. 2011.

12. Dyerberg J, Madsen P, Møller JM, Aardestrup I, Schmidt EB. Bioavailability of marine $n-3$ acid formulations. Prostaglandins leukotrienes essential fatty acids. 2010. 83: 137-141. Ref.: https://goo.gl/RpJrSL

13. Siscovick DS, Raghunathan TE, King I, Weinmann S, Wicklund KG, et al. Dietary Intake and Cell Membrane Levels of Long Chain Omega-3 Polyunsaturated Fatty Acids and the Risk of Primary Cardiac Arrest. JAMA. 1995; 274: 1363-1367. Ref.: https://goo.gl/9WC4JA 
14. The definition and classification of dry eye disease: report of the Definition and Classification Subcommittee of the International Dry Eye WorkShop (2007). Ocul Surf. 2007; 5: 75-92. Ref.: https://goo.gl/Zxzocb

15. Nichols KK. The International Workshop on Meibomian gland Dysfunction. Invest Ophthalmol Vis Sci. 2011: 52: 1917-1921. Ref.: https://goo.gl/yr5m1t

16. Swanson D, Block R, Mousa SA. Omega-3 fatty acid EPA and DHA: Health Benefits throughout life. Adv Nutr. 2012; 3: 1-7. Ref.: https://goo.gl/fupSE4

17. Deckelbaum RJ, Torrejon C. The Omega-3 fatty acid nutritional landscape: Health Benefits and sources. J Nutr. 2012; 142: 587-591. Ref.: https://goo.gl/PUkswP

18. Pinna A, Blasetti F, Zinellu A, Carru C, Solinas G. Meibomian Gland Dysfunction and Hypercholesterolemia. Ophthalmology. 2013; 120: 2385-2389. Ref.: https://goo.gl/1dVLfu

19. Sala-Vila A, Díaz-López A, Valls-Pedret C, Cofán M, García-Layana A, et al. Dietary Marine Omega-3 Fatty Acids and Incident Sight Threatening Retinopathy in Middle Aged and Older Individuals with Type 2 Diabetes. JAMA Ophthalmol. 2016; 134: 1142-1149. Ref.: https://goo.gl/AjtE4s

20. Abelson MB, McLaughlin J. "Resolving" The effects of Fish Oil on the eye. Rev. Ophth. 2012; 74-77.

21. Epitropoulos AT, Donnenfeld ED, Shah ZA, Holland EJ, Faulkner WJ, et al. Effect of Oral ReEsterified Omega-3 Nutritional Supplementation on Dry Eyes. Cornea. 2016; 35: 1185-1191. Ref.: https://goo.gl/P6apTE

22. Danaei G, Ding EL, Mozaffarian D, Taylor B, Rehm J, et al. The Preventable Cause of Death in the United Sates: Comparative Risk Assessment of Dietary, Lifestyle and Metabolic Risk Factors. PLOS Med. 2009. Ref.: https://goo.gl/Cd2fBE

23. Brasky TM, et al., Nutr Cancer, 2011, doi: 10.1080/01635581.2011.553022

24. White D, Study sends wrong message about Omega-3 fatty acids and prostate cancer. Ocular Surgery News, 3/25/2014, 8 .

25. Chinnery HR, Naranjo Golborne C, Downie LE. Omega-3 supplementation is neuroprotective to corneal nerves in dry eye disease: a pilot study. Ophthalmic Physiol Opt. 2017; 37: 473-481. Ref.: https://goo.gl/EwvBXW

26. Bhargava R, Mini Chandra, Utsav Bansal, Divya Singh, Somesh Ranjan. A Randomized Controlled Trial of Omega 3 Fatty Acids in Rosacea Patients with Dry Eye Symptoms. Current Eye Research. 2016. https://goo.gl/oH5PzU

27. Gatell-Tortajada J. Oral supplementation with a nutraceutical formulation containing omega-3 fatty acids, vitamins, minerals, and antioxidants in a large series of patients with dry eye symptoms: results of a prospective study. Clin Interv Aging. 2016; 11: 571-578. Ref.: https://goo.gl/8JUrAL

28. Molina-Leyva I, Molina-Leyva A, Bueno-Cavanillas A. Efficacy of nutritional Supplementation with omega-3 and omega- 6 fatty acids in dry eye syndrome: a systematic review of randomized clinical trials. Acta Ophthalmol.2017; 95: 677-685. Ref.: https://goo.gl/FhTnfj

29. Age-Related Eye Disease Study Protocol. 